\title{
Leptomeningeal Carcinomatosis: A Call for Optimizing Diagnostic Sensitivity by the Hematology Laboratory
}

\author{
Benjamin Lardinois ${ }^{a} \quad$ Laurence Miller $^{a} \quad$ Adrien Randazzo ${ }^{a}$ Terry Laurent ${ }^{a}$ \\ Régis Debois ${ }^{a}$ Stéphanie Henry ${ }^{b}$ \\ aDepartment of Laboratory, CHU UCL Namur Site de Sainte-Elisabeth, Université Catholique \\ de Louvain, Namur, Belgium; 'bepartment of Oncology, CHU UCL Namur Site de Sainte- \\ Elisabeth, Université Catholique de Louvain, Namur, Belgium
}

\author{
Keywords \\ Leptomeningeal carcinomatosis $\cdot$ Cytology $\cdot$ Breast cancer
}

\begin{abstract}
In the cerebrospinal fluid (CSF), the demonstration of malignant cells by cytological examination is currently the gold standard for the diagnosis of leptomeningeal carcinomatosis (LC). However, a positive cytology is observed in only $50-60 \%$ of patients with LC and highly dependent on pre-analytical factors. The hematology laboratory could provide an immediate and accurate diagnosis, but diagnostic sensitivity is not always optimized once the sample is received. We hereby report a 49-year-old woman with a 3-year grade III invasive ductal carcinoma who was admitted to the emergency department due to headaches, nausea, and vomiting. The CSF revealed pleocytosis with suspicious high fluorescent cells on the hematology analyzer concomitantly with biochemical alterations. Cytomorphological examination confirmed tumor cells, thus diagnosing a leptomeningeal metastasis of her breast cancer. The patient was eventually transferred to palliative care. Cytological examination is a valuable tool for a rapid diagnosis of LC if diagnostic performance is optimized. In addition to repeated CSF collections with a sufficient volume $(5-10 \mathrm{~mL})$, this could be reached by processing the CSF as soon as possible, taking into account the fluorescence information from the analyzer, proceeding systematically to microscopic examination even with normal CSF white blood cell count, and providing quality improvement of the staff to identify malignant cells.
\end{abstract}

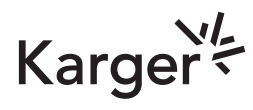


Lardinois et al.: The Importance of Cytology in Leptomeningeal Carcinomatosis

\section{Introduction}

Brain metastasis is a relatively common complication of patients with advanced breast cancer occurring in 10-15\% of cases [1]. These central nervous system metastases can infiltrate the leptomeninges, the brain parenchyma, or both [2]. Leptomeningeal dissemination is an estimated complication occurring in up to $5 \%$ of breast cancer patients [3] and refers to tumor infiltration of the CSF and leptomeninges. It causes high morbidity and poor prognosis, and therefore CSF analyses should be carried out once suggestive clinical signs are observed [4]. Although clinical manifestations and neuroimaging findings are valuable tools in the clinical assessment, the cytological examination is currently considered as the gold standard for the diagnosis of LC but is only positive in $50-60 \%$ of patients, thus forcing clinicians to repeat lumbar punctures up to 3 times or even sometimes consider alternative diagnosis [5]. This low diagnostic sensitivity is mainly explained by exacting pre-analytical conditions, including a quick processing of the CSF specimen [6] and a sufficient volume for analysis [4]. In this report, we described a patient with metastatic breast cancer admitted for neurological manifestations, without specific radiological findings but a positive CSF cytology revealing the presence of neoplastic cells. It emphasizes the crucial importance of the laboratory for the detection of malignant cells in CSF by illustrating and identifying the conditions to be optimized.

\section{Case Presentation}

A 49-year-old woman with a 3-year, grade III, triple-negative, invasive ductal carcinoma was admitted to the emergency department due to headaches, nausea, and vomiting. She was previously known to have liver metastases and was weekly treated with gemcitabine and carboplatin.

A previous follow-up MRI had shown a supratentorial metastatic lesion, but no abnormal cells were detected in the CSF. This metastasis had been completely resected, and histopathology confirmed a triple-negative adenocarcinoma with a Ki-67 proliferative index of approximately 99\%. Brain and breast MRI follow-ups had shown no specific evolution. Two months after the surgery, facial nerve invasion was suspected, and a pan-encephalic radiotherapy was delivered. The cancer antigen 15-3 had been fluctuating between 30 and $60 \mathrm{U} /$ $\mathrm{mL}(\mathrm{NR}<30 \mathrm{U} / \mathrm{mL})$ since 8 months and slightly decreasing since 2 months.

On admission, the patient had CSF alterations including mild pleocytosis at $0.024 \times 10^{9} / \mathrm{L}$ (normal range $[\mathrm{NR}]<0.005 \times 10^{9} / \mathrm{L}$ ), elevated protein at $1.33 \mathrm{~g} / \mathrm{L}(\mathrm{NR} 0.10-0.45 \mathrm{~g} / \mathrm{L}$ ), and high lactate at $8.71 \mathrm{mmol} / \mathrm{L}$ (NR 1.20-2.10 mmol/L). Hypoglycorrhachia was also observed with cerebrospinal glucose measured at $0.55 \mathrm{mmol} / \mathrm{L}$ (NR 2.5-4.44 mmol/L). An MRI carried out 3 days before admission had shown no particular lesions. Interestingly, the body fluid scattergram of the Sysmex XN-1000 hematology analyzer (Sysmex, Kobe, Japan) exhibited suspicious cells with high fluorescence, bigger than the monocyte cluster (shown in Fig. 1) with a HF-BF\% of $100 \%$ and a HF-BF count of $0.024 \times 10^{9} / \mathrm{L}$ (no cutoff available). CSF cytomorphologic investigation by the hematology laboratory subsequently confirmed the presence of malignant cells with typical abnormalities after a cytospin and May-GrunwaldGiemsa staining method, which was highly indicative of leptomeningeal carcinomatosis (LC) (shown in Fig. 2). Subsequent immunohistochemical staining by the histopathology laboratory including CAM 5.2 and GATA3 was noncontributory, but direct morphological examination also confirmed a carcinomatosis of mammary origin. After careful prognostic evaluation and multidisciplinary discussion, the patient eventually received eribulin and was, in agreement with the family, transferred to palliative care.

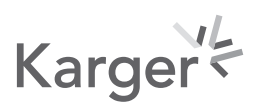




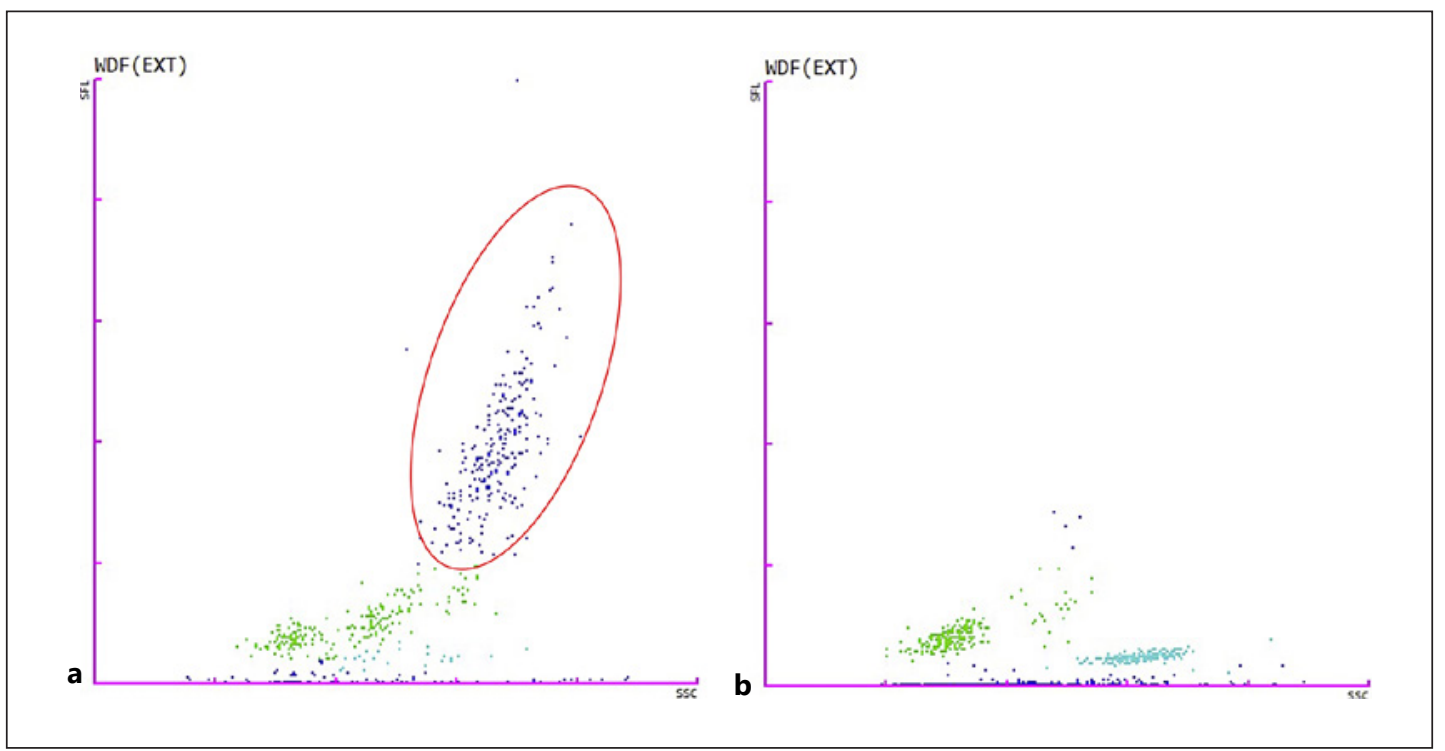

Fig. 1. Body fluid scattergrams: WDF scattergram of the patient's cerebrospinal carcinomatous fluid showed high fluorescent cells (HF-BF\%: 100\%) (A) compared to a CSF with a similar WBC count in a patient without leptomeningeal carcinomatosis (HF-BF\%: 2.3\%) (B). The greater dispersion of these cells reflects a wide heterogeneity of nucleic acid content and internal cell structure (red ellipse). SFL, side fluorescence; SSC, side scatter; WDF, WBC differential fluorescence; CSF, cerebrospinal fluid.

\section{Discussion}

The search for neoplastic cells in a fluid is commonplace in a clinical laboratory. However, there are few expert recommendations concerning the pre-analytical phase, and the interpretation is based on the experience of the cytologist. Many doubtful cases remain inconclusive, and histologic examination is often late. In the CSF, the demonstration of malignant cells by cytological examination is the accepted gold standard for the diagnosis of LC but exhibits low sensitivity [7]. False-negative results exist despite repeated examinations, in particular due to a too low volume or delays before processing [4]. Interestingly, a study in 2018 showed that positive CSF cytology was more frequent in patients with breast cancer and adenocarcinoma than those with small-cell lung cancer [8]. In addition, CSF biochemical alterations could be observed in $90 \%$ of the patients with leptomeningeal disease [4].

Due to that, hematology laboratories should optimize their detection rate and diagnostic sensitivity by carrying out the following steps:

1. Immediately processing the CSF specimen to avoid in vitro time-dependent cell death [6].

2. Taking into account the fluorescence information if provided by the analyzer (e.g., XN series [Sysmex], UniCel DxH [Beckman Coulter, Brea, CA, USA] or UF-5000 [Sysmex]) as it is clinically advantageous when combined with optical microscopy and shows good analytical performance $[9,10]$. Further studies are however needed to correlate fluorescence intensity with cancer type and/or histological subtype although a better harmonization of cytometric analysis in body fluid samples should be preferentially achieved.

3. Proceeding mandatorily to a CSF microscopic examination, even when a normal CSF white blood cell count is observed or MRI is negative as it does not exclude leptomeningeal metastasis [11]. 

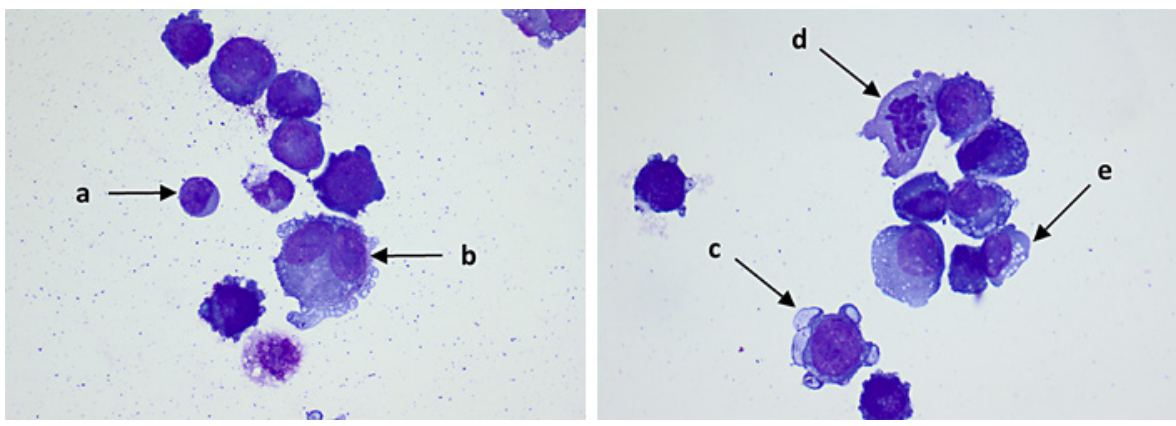

Fig. 2. Morphology: cytomorphological analysis on CSF was carried out after a cytospin and May-GrunwaldGiemsa staining method. It highlighted giant basophilic cells compared to a normal lymphocyte (A). Multinuclearity with uncondensed nuclear chromatin and large angulated nucleoli (B), vesicle-like cytoplasmic membrane projections (C), asymmetrical mitotic figure (D), and cytoplasmic vacuoles with some over the nucleus (E) were observed, thus arguing for metastatic carcinomatous cells. Magnification, $\times 100$. CSF, cerebrospinal fluid.

4. Providing quality improvement of the hematology staff in identifying cell morphology. This could reach a close malignancy detection rate to the histology laboratory $[12,13]$. This is especially justified given that histopathology is not always requested.

A standardized cytological terminology should also be applied in CSF as inflammatory cells with atypical morphology could be observed, and some cases remain doubtful. Indeed, specific terminology has recently been defined by an expert consensus but restricted to serous fluid cytology. The authors suggested 5 cytopathologic diagnostic categories, each associating a risk of malignancy in order to minimize the use of atypical and suspicious diagnoses [14]. For example, a class 5 category makes a definitive diagnosis of malignancy, similar to histopathological examinations. It could therefore provide a degree of diagnostic certainty to oncologists. Applied in the patient's CSF, the typical cellular abnormalities observed were category 5 , thus affirming the diagnosis of LC.

In the absence of malignant cells detected in the CSF, concomitant clinical symptoms and typical MRI findings may also establish a definitive diagnosis of LC [15]. However, except for aspecific neurological symptoms, our patient did not present any radiological findings either, and only later histopathological results would have allowed the diagnosis if cytology was reported negative.

Future algorithms including clinical information, radiological findings, and standardized cytology will soon further assess the risk of LC and patient prognosis. Finally, the need for high diagnostic accuracy on CSF specimens is crucial to limit invasive additional examinations, costs, and prolongation of hospital stay.

\section{Conclusion}

LC has a poor prognosis and requires a diagnosis as soon as patients manifest clinical signs. Although considered as the gold standard to diagnose LC, CSF cytology significantly lacks diagnostic sensitivity. As presented in this case, neuroimaging may also be inconclusive. Therefore, several factors identified in the literature must now be taken into account to maximize the chances of detecting tumor cells. In addition to repeated CSF collections with a sufficient volume (5-10 mL), these include quickly processing the specimen once arrived in the laboratory, integrating the fluorescence information in the diagnostic evaluation if provided by the analyzer, proceeding systematically to microscopic examination even with 
normal CSF white blood cell count, and providing quality improvement of the staff to identify malignant cells. Taking these considerations into account will limit the time to treatment, the associated morbidity, the additional (invasive) examinations, and the overall cost of care.

\section{Acknowledgment}

The authors thank the laboratory staff for processing the analysis.

\section{Statement of Ethics}

Written informed consent was obtained from the patient's next of kin for publication of this case report and any accompanying images. Our institution does not require ethics committee approval for a case report.

\section{Conflict of Interest Statement}

The authors have no conflicts of interest to declare.

\section{Funding Sources}

This research received no external funding.

\section{Author Contributions}

B.L., L.M., A.R., L.T., R.D., and S.H. analyzed and interpreted the biological and oncological data and read and approved the final manuscript. B.L. contributed to the literature review, manuscript writing, and submission.

\section{Data Availability Statement}

The data that support the findings of this study are not publicly available because they contain information that could compromise the patient's privacy but are available from the corresponding author (B.L.).

\section{References}

1 Barnholtz-Sloan JS, Sloan AE, Davis FG, Vigneau FD, Lai P, Sawaya RE. Incidence proportions of brain metastases in patients diagnosed (1973 to 2001) in the metropolitan detroit cancer surveillance system. J Clin Oncol. 2004 Jul 15;22(14):2865-72.

2 Sekhar A, Corbo B, Das K, Biswas S. Leptomeningeal carcinomatosis: easy to miss. J R Coll Physicians Edinb. 2017;47(4):351-2.

3 Corbin ZA, Nagpal S. Leptomeningeal metastases. JAMA Oncol. 2016 Jun 1;2(6):839.

4 Franzoi MA, Hortobagyi GN. Leptomeningeal carcinomatosis in patients with breast cancer. Crit Rev Oncol Hematol. 2019 Mar;135:85-94.

\section{Karger's}


5 Weston CL, Glantz MJ, Connor JR. Detection of cancer cells in the cerebrospinal fluid: current methods and future directions. Fluids Barriers CNS. 2011;8(1):14.

6 Wang N, Bertalan MS, Brastianos PK. Leptomeningeal metastasis from systemic cancer: review and update on management: management of leptomeningeal metastasis. Cancer. 2018 Jan 1;124(1):21-35.

7 Lee JS, Melisko ME, Magbanua MJ, Kablanian AT, Scott JH, Rugo HS, et al. Detection of cerebrospinal fluid tumor cells and its clinical relevance in leptomeningeal metastasis of breast cancer. Breast Cancer Res Treat. 2015 Nov;154(2):339-49.

8 Pan Z, Yang G, He H, Yuan T, Wang Y, Li Y, et al. Leptomeningeal metastasis from solid tumors: clinical features and its diagnostic implication. Sci Rep. 2018 Dec;8(1):10445.

9 Buoro S, Seghezzi M, Dominoni P, Moioli V, Manenti B, Previtali G, et al. Lack of harmonization in high fluorescent cell automated counts with body fluids mode in ascitic, pleural, synovial, and cerebrospinal fluids. Int J Lab Hematol. 2019 Apr;41(2):277-86.

10 Cho J, Oh J, Lee SG, Lee YH, Song J, Kim JH. Performance evaluation of body fluid cellular analysis using the Beckman Coulter UniCel DxH 800, Sysmex XN-350, and UF-5000 automated cellular analyzers. Ann Lab Med. 2020 Mar 1;40(2):122-30.

11 Bönig L, Möhn N, Ahlbrecht J, Wurster U, Raab P, Puppe W, et al. Leptomeningeal metastasis: the role of cerebrospinal fluid diagnostics. Front Neurol. 2019 Aug 20;10:839.

12 Li X, Zhang Y, Ding J, Wang M, Li N, Yang H, et al. Clinical significance of detecting CSF-derived tumor cells in breast cancer patients with leptomeningeal metastasis. Oncotarget. 2018 Jan 5;9(2):2705-14.

13 Zhang ML, Maglantay RJ, Cunningham VL, Goodwin MT, Feeney MW, Keefe J, et al. Improving malignancy detection rates in body fluids submitted to the hematology laboratory for nucleated cell count and differential: a quality improvement study. Arch Pathol Lab Med. 2021 Feb 1;145(2):201-7.

14 Pinto D, Chandra A, Crothers BA, Kurtycz DFI, Schmitt F. The international system for reporting serous fluid cytopathology-diagnostic categories and clinical management. J Am Soc Cytopathol. 2020 Nov; 9(6):469-77.

15 Le Rhun E, Weller M, Brandsma D, Van den Bent M, de Azambuja E, Henriksson R, et al. EANO-ESMO Clinical Practice Guidelines for diagnosis, treatment and follow-up of patients with leptomeningeal metastasis from solid tumours. Ann Oncol. 2017 Jul;28(Suppl 4):iv84-99. 